\title{
ON THE NONCENTRAL DISTRIBUTION OF THE RATIO OF THE EXTREME ROOTS OF THE WISHART MATRIX
}

\author{
V.B. WAIKAR \\ Department of Mathematics \& Statistics \\ Miami University \\ Oxford, Ohio 45056 \\ USA
}

(Received February 6, 1979)

ABSTRACT. The distribution of the ratio of the extreme latent roots of the Wishart matrix is useful in testing the sphericity hypothesis for a multivariate normal population. Let $\mathrm{X}$ be a $\mathrm{p} x \mathrm{n}$ matrix whose columns are distributed independently as multivariate normal with zero mean vector and covariance matrix $\sum$. Further, let $S=X X^{\prime}$ and 1 et $1_{1}>\ldots>1_{p}>0$ be the characteristic roots of $S$. Thus $\mathrm{S}$ has a noncentral Wishart distribution. In this paper, the exact distribution of $f_{p}=1-1_{p} / 1_{1}$ is derived. The density of $f_{p}$ is given in terms of zonal polynomials. These results have applications in nuclear physics also. KEY WORDS AND PHRASES. Extreme roots, Wishart distribution, Zonal polynomials 1980 MATHEMATICS SUBJECT CLASSIFICATION CODES. Primary 62H10; Secondary 62 E15.

1. INTRODUCTION.

The distribution of the ratio of the extreme latent roots of the Wishart 
matrix is useful in testing the sphericity hypothesis for a multivariate normal population. In the central (null) case, Sugiyama (1970) derived the density of the ratio of the smallest to the largest root of the Wishart matrix when the associated covariance matrix is the identity matrix. Waikar and Schuurmann (1973) derived an alternate expression which is much superior to that given by Sugiyama (1970) from the point of view of computing and in fact we computed some tables of the percentage points which are also included in the above paper. In this paper, the author has derived an exact expression for the ratio of the smallest to the largest root of the noncentral Wishart matrix. This research has applications in nuclear physics [see Wigner (1967)].

Constantine [1, p. 1277] defines the non-central Wishart distribution. Anderson [2, p. 409] relates the Wishart and non-central Wishart distributions to each other and to neighboring areas of multivariate analysis. James [3, p.475] gives a brief exposition of this area of multivariate analysis.

\section{PRELIMINARIES.}

If $\mathrm{A}$ is a square, nonsingular matrix its inverse and determinant are denoted respectively by $A^{-1}$ and $A \mid$ The transpose, trace and exponential of the trace of a matrix $B$ are denoted respectively by $B^{\prime}$, tr $B$ and etr $B$. Also $I_{p}$ and $0_{p}$ denote respectively a $\mathrm{p} x \mathrm{p}$ identity matrix and a $\mathrm{p} x \mathrm{p}$ null matrix. In addition, we define as in James (1964).

$$
\begin{aligned}
& p_{q}{ }_{q}\left(a_{1}, \ldots, a_{p} ; b_{1}, \ldots, b_{q} ; s\right) \\
& =\sum_{k=0}^{\infty} \sum_{K} \frac{\left(a_{1}\right)_{K} \cdots\left(a_{p}\right)_{K}}{\left(b_{1}\right)_{K} \cdots\left(b_{q}\right)_{K}} \frac{c_{K}(s)}{k !}
\end{aligned}
$$

and

$$
\begin{aligned}
& p_{q}^{F}\left(a_{1}, \ldots, a_{p} ; b_{1}, \ldots, b_{q} ; s, T\right) \\
= & \sum_{k=0}^{\infty} \sum_{K} \frac{\left(a_{1}\right)_{K} \cdots\left(a_{p}\right)_{K}}{\left(b_{1}\right)_{K} \cdots\left(b_{k}\right)_{K}} \frac{c_{K}(T)}{c_{K}\left(I_{p}\right) k !}
\end{aligned}
$$


where $S$ and $T$ are $p \times p$ symetric matrices and $k=\left(k_{1}, \ldots, k_{p}\right)$ is a partition of the integer $k$ satisfying (i) $k_{1} \geq k_{2} \geq \cdots \geq k_{p} \geq 0$ and (ii) $k_{1}+\ldots+k_{p}=k$. Further

$$
\begin{gathered}
(a)_{k}=\prod_{i=1}^{p}(a-(i-1) / 2)_{k_{i}} \\
(a)_{k}=a(a+1) \ldots(a+1-k), \quad(a)_{0}=1
\end{gathered}
$$

and finally $C_{K}(S)$ is the zonal polynomial as defined in James (1964) and satisfies $(\operatorname{tr} S)^{k}=\sum C_{K}(S)$. A special case of the above is

$$
{ }_{1} F_{0}(a ; S)^{k}=\left|I_{p}-S\right|^{-a} \text {. }
$$

\section{REMARK 1.}

Note that if one of the $a_{i}$ 's above is a negative integer say $a_{1}=-n$ then for $k \geq p n+1$ all the coefficients vanish so that the function $p^{F} q$ reduces to a (finite) polynomial of degree pn (see Constantine (1963) p. 1276). Further, throughout the paper, whenever a partition say $k=\left(k_{1}, \ldots, k_{p}\right)$ of a nonnegative integer $k$ is defined, it will be implied that

$$
\text { (i) } k_{1} \geq \ldots \geq k_{p} \geq 0 \text { and (ii) } k_{1}+\ldots+k_{p}=k
$$

The following three lemmas are needed in the sequel.

LEMMA 2.1. Let $k$ and $d$ be two nonnegative integers and let $k=\left(k_{1}, \ldots, k_{p}\right)$ and $\delta=\left(d_{1}, \ldots, d_{p}\right)$ denote partitions respectively of $k$ and $d$. Further let $G=$ $\operatorname{diag}\left(g_{1}, \ldots, g_{p}\right)$. Then

$$
C_{K}(G) \cdot C_{\delta}(G)=\sum_{\dot{\beta}} g_{K, \delta}^{\beta} C_{\beta}(G)
$$

where $\beta=\left(b_{1}, \ldots, b_{p}\right)$ is a partition of the integer $k+d=b$.

LEMMA 2.2. Let $G$ be as defined in Lemma 2.1 and further let $G_{1}=\operatorname{diag}(1, G)$. Let $k=\left(k_{1}, \ldots, k_{p+1}\right)$ be a partition of a nonnegative integer $k$. Then

$$
C_{K}\left(G_{1}\right)=\sum_{t=0}^{\kappa} \sum_{\tau} b_{k, \tau} C_{\tau}(G)
$$


where $\tau=\left(t_{1}, \ldots, t_{p}\right)$ is a partition of $t$.

The above two lemmas are stated in Khatri and Pillai (1968). The g-coefficients in (2.1) and the b-coefficients in (2.2) were tabulated by Khatri and Pillai (1968) for various values of the arguments and can be obtained from them.

Throughout this paper, the following notations will be used:

$$
\begin{aligned}
& \Gamma_{p}(a)=\pi^{p(p-1) / 4} \prod_{i=1}^{p} \Gamma(a-(i-1) / 2) \\
& \underset{i<j=t}{p}\left(a_{i}-a_{j}\right)=\prod_{i=t}^{p-1} \prod_{j=i+1}^{p}\left(a_{i}-a_{j}\right), 0 \leq t \leq p .
\end{aligned}
$$

The following lemma can be proved by making trivial modification in the proof of the Lemma given in Sugiyama (1967).

LEMMA 2.3. Let $R=\operatorname{diag}\left(r_{1}, \ldots, r_{p-1}\right)$ where $0<r_{1}<\ldots<r_{p-1}<1$ and let $\mathrm{R}_{1}=\operatorname{diag}\left(\mathrm{r}_{1}, \ldots, \mathrm{r}_{\mathrm{p}-1}, 1\right)$. Further let $k=\left(\mathrm{k}_{1}, \ldots, \mathrm{k}_{\mathrm{p}}\right)$ be a partition of the positive integer $k$. Then

$$
\begin{aligned}
D_{K}^{\mathrm{P}}(t) & =\int_{0<r_{1}<\cdots<x_{p-1}<1} \ldots \iint_{R}^{t-(p+1) / 2}\left|I_{p-1}-R\right| C_{K}\left(R_{1}\right) \prod_{i>j=1}^{p-1}\left(r_{i}-r_{j}\right) d R \\
& =(p t+k)\left(\Gamma_{p}(p / 2) / \pi^{p^{2} / 2}\right) C_{K}\left(I_{p}\right)\left(\Gamma_{p}(t, k) \Gamma_{p}((p+1) / 2)\right) / \Gamma_{p}(t+(p+1) / 2, k)
\end{aligned}
$$

where

$$
\Gamma_{p}(a, k)=\pi^{p(p-1) / 4} \underset{i=1}{p} \Gamma\left(a+k_{i}-(i-1) / 2\right) .
$$

LEMMA 2.4. Let $A$ be any $p \times p$ matrix and let $k=\left(k_{1}, \ldots, k_{p}\right)$ be a partition of a nonnegative integer $k$. Then

$$
C_{k}\left(I_{p}+A\right)=\sum_{g=0}^{k} \sum_{\gamma} a_{k, \gamma} C_{\gamma}(A) C_{k}\left(I_{p}\right) / C_{\gamma}\left(I_{p}\right),
$$

where $\gamma=\left(g_{1}, \ldots, g_{p}\right)$ is a partition of $g$.

The above lemma is stated in Constantine (1963) and some tabulations of acoefficients are also given in the same paper. 
3. DENSITY OF THE RATIO OF THE SMALLEST TO THE LARGEST ROOT OF THE WISHART MATRIX.

Let $\mathrm{X}$ be a $\mathrm{p} x \mathrm{n}$ matrix whose columns are distributed independently as multivariate normal with zero mean vector and covariance matrix $\sum$ and let $\mathrm{p} \leq \mathrm{n}$. Further, let $S=X X^{\prime}$ and let $\ell_{1}>\ell_{2}>\ldots>\ell_{p}>0$ be the characteristic roots of $S$. Thus $\mathrm{S}$ has a noncentral Wishart distribution and the joint density of its roots $\ell_{1}, \ldots, \ell_{\mathrm{p}}$ as derived by James (1964) is

$h_{1}\left(l_{1}, \ldots, l_{p}\right)$

$=\left.\mathrm{k}(\mathrm{p}, \mathrm{n})\left|\Sigma^{-\mathrm{n} / 2}{ }_{0} \mathrm{~F}_{0}\left(\mathrm{I}-\frac{1}{2} \Sigma^{-1}, \mathrm{~L}\right) \operatorname{etr}(-\mathrm{L})\right| \mathrm{L}\right|^{(\mathrm{n}-\mathrm{p}-1) / 2}$

$$
\times \prod_{i<j=1}^{p}\left(\ell_{i}-\ell_{j}\right), \infty>\ell_{1}>\ldots>\ell_{p}>0
$$

where $L=\operatorname{diag}\left(\ell_{1}, \ldots, l_{p}\right)$ and $k(p, n)=\Pi^{p^{2} / 2} /\left(2^{p n / 2} \Gamma_{p}(n / 2) \Gamma_{p}(p / 2)\right)$. Now, on making the transformation $\ell_{1}=\ell_{1}, f_{i}=1-\ell_{i} / l_{1} i=2, \ldots, p$ in $(3.1)$, we obtain the joint density of $\ell_{1}, \mathrm{f}_{2}, \ldots, \mathrm{f}_{\mathrm{p}}$ as

$h_{2}\left(l_{1}, f_{2}, \ldots, f_{p}\right)$

$=k(p, n)\left|\sum\right|^{-n / 2} \ell_{1}^{p(n-2) / 2} e^{-p^{\ell} 1} \operatorname{etr}\left(\ell_{1} F\right)|F|$ $\left|I_{p-1}-F\right|^{(n-p-1) / 2} \prod_{i>j=2}^{p}\left(f_{i}-f_{j}\right)$

$$
\begin{aligned}
& \sum_{k=0}^{\infty} \sum_{K} \frac{C_{K}\left(I-\frac{1}{2} \sum^{-1}\right) \ell_{1}^{k}}{k ! C_{K}\left(I_{p}\right)} C_{K}\left[\operatorname{diag}\left(1, I_{p-1}-F\right)\right] \\
& 0<\ell_{1}<\infty, 0<f_{2}<\ldots<f_{p}<1
\end{aligned}
$$

where $F=\operatorname{diag}\left(f_{2}, \ldots, f_{p}\right)$ and $k=\left(k_{1}, \ldots, k_{p}\right)$ is a partition of the integer $k$. On using Lemmas 2.2 and 2.4 to expand $C_{K}[\operatorname{diag}(1, I-F)]$, and further, writing etr $\left(\ell_{1} F\right)$ as ${ }_{0} F_{0}\left(l_{1} F\right)$ and then expanding it we can rewrite the above density as $h_{3}\left(l_{1}, f_{2}, \ldots, f_{p}\right)=$ 
$=k(p, n)|\Sigma|^{-n / 2} e_{1}^{p(n-2) / 2} e^{-p l_{1}}|F||I-F|^{(n-p-1) / 2}$

$$
\prod_{i>j=2}^{p}\left(f_{i}-f_{j}\right) \sum_{k=0}^{\infty} \sum_{k} \frac{C_{k}\left(I-\frac{1}{2} \sum^{-1}\right) l_{1}^{k}}{k ! C_{k}(I)} \sum_{a=0}^{\infty} \sum_{\alpha} \frac{l_{1}^{a} C_{\alpha}(F)}{a !}
$$

$\sum_{t=0}^{k} \sum_{\tau} b_{k, \tau} \sum_{g=0}^{t} \sum_{\gamma} \frac{a_{\tau}, \gamma c_{\gamma}(-F) c_{\tau}(I)}{C_{\gamma}(I)}, 0<\ell_{1}<\infty, 0<f_{2}<\ldots<f_{p}<1$

where $\alpha=\left(a_{1}, \ldots, a_{p-1}\right)$ is a partition of the integer $a, \tau=\left(t_{1}, \ldots, t_{p-1}\right)$ is a partition of the integer $t, \gamma=\left(g_{1}, \ldots, g_{p-1}\right)$ is a partition of $g$ and the $b_{k, \tau}$ and $a_{\tau, \gamma}$ are given by Lemmas 2.2 and 2.4 respectively. Now note that $C_{\alpha}(F) C_{\gamma}(-F)=\sum_{\eta}(-1)^{g} g_{\alpha, \gamma}^{\eta} C_{\eta}(F)$

where $n=\left(n_{1}, \ldots, n_{p-1}\right)$ is a partition of $a+g$ and the coefficients $g_{\alpha, \gamma}^{n}$ are given by Lemma 2.1. Further

$$
\begin{aligned}
& |I-F|^{(n-p-1) / 2}={ }_{1} F_{0}(-(n-p-1) / 2, F) \\
& =\sum_{d=0}^{\infty} \sum_{\delta} \frac{(-(n-p-1) / 2)}{d !} \delta c_{\delta}(F)
\end{aligned}
$$

where $\delta=\left(d_{1}, \ldots, d_{p-1}\right)$ is a partition of the integer $d$.

Also then $C_{\eta}(F) C_{\delta}(F)=\sum_{\beta} g_{\eta, \delta}^{\beta} C_{\beta}(F)$

where $\beta=\left(b_{1}, \ldots, b_{p-1}\right)$ is a partition of $(a+g)+d$ and the coefficients $g_{n, \delta}^{\beta}$ are given by Lemma 2.1. Thus the density in (3.3) becomes

$h_{4}\left(l_{1}, f_{2}, \ldots, f_{p}\right)$

$$
\begin{aligned}
= & k(p, n)|\Sigma|^{-n / 2} \sum_{k=0}^{\infty} \sum_{k} \frac{c_{k}\left(I-\frac{1}{2} \sum^{-1}\right)}{k ! c_{k}(I)} \sum_{a=0}^{\infty} \sum_{\alpha} \sum_{t=0}^{k} \sum_{\tau} \frac{b_{k, \tau}}{a !} \\
& \sum_{g=0}^{t} \sum_{\gamma} \frac{a_{\tau, \gamma} C_{\tau}(I)(-1)^{g}}{c_{\gamma}(I)} \sum_{n} g_{\alpha, \gamma}^{n} \sum_{d=0}^{\infty} \sum_{\delta} \frac{(-(n-p-1) / 2)_{\delta}}{d !}
\end{aligned}
$$




$$
\begin{aligned}
\sum_{\beta} g_{n, \delta}^{\beta} l_{1}^{p(n-2) / 2+k+a} & e^{-p l_{1}}|F| \underset{i>j=2}{p}\left(f_{i}-f_{j}\right) c_{\beta}(F), \\
& 0<l_{1}<\infty, 0<f_{2}<\ldots<f_{p}<1 .
\end{aligned}
$$

Now, on making the transformation $\ell_{1}=\ell_{1}, r_{i}=f_{i} / f_{p}, i=2, \ldots, p-1, f_{p}=f_{p}$ and then integrating out $r_{2}, \ldots, r_{p-1}$ over the surface $0<r_{2}<\ldots<r_{p-1}<1$ (using Lemma 2.3) we get the joint density of $l_{1}$ and $f_{p}$ as

$$
\begin{aligned}
& h_{5}\left(l_{1}, f_{p}\right)=k(p, n)\left|\sum\right|^{-n / 2} \sum_{k=0}^{\infty} \sum_{k} \frac{C_{k}\left(I-\frac{1}{2} \Sigma^{-1}\right)}{k ! C_{K}(I)} \\
& \sum_{a=0}^{\infty} \sum_{\alpha} \sum_{t=0}^{k} \sum_{\tau} \frac{b_{K, I}}{a !} \sum_{g=0}^{t} \sum_{\gamma} \frac{a_{\tau, \gamma} C_{\tau}(I)(-1)^{g}}{C_{\gamma}(I)} \\
& \sum_{n} g_{\alpha, \gamma}^{n} \sum_{d=0}^{\infty} \sum_{\delta} \frac{(-(n-p-1) / 2) \delta}{d !} \sum_{\beta} g_{i n, \delta}^{\beta} \\
& \ell_{1}^{p(n-2) / 2+k+a} e^{-p l} e_{p}^{\frac{1}{2}} f^{p(p+1)-2+a+g+d} D_{\beta}^{p-1}\left(\frac{p+2}{2}\right), \\
& 0<\ell_{1}<\infty, 0<f_{p}<1
\end{aligned}
$$

where $D_{\beta}^{\mathrm{p}-1}((\mathrm{p}+2) / 2)$ is given by Lemma 2.3 .

Now, on integrating out $\ell_{1}\left(0<\ell_{1}<\infty\right)$, we get the marginal density of $f_{p}=1-\ell_{p} / l_{1}$ as

$$
\begin{aligned}
& h_{6}\left(f_{p}\right)=k(p, n)\left|\sum\right|^{-n / 2} \sum_{k=0}^{\infty} \sum_{k} \frac{c_{k}\left(I-\frac{1}{2} \sum^{-1}\right)}{k ! C_{k}(I)} \\
& \sum_{a=0}^{\infty} \sum_{\alpha} \sum_{t=0}^{k} \sum_{\tau} \frac{b_{k, \tau}}{a !} \sum_{g=0}^{\tau} \sum_{\gamma} \frac{a, \gamma_{\tau} C_{\tau}(I)(-1)^{g}}{C_{\gamma}(I)} \\
& \sum_{n} g_{\alpha, \gamma}^{n} \sum_{\alpha=0}^{\infty} \sum_{\delta} \frac{(-(n-p-1) / 2) \delta_{\delta}}{d !} \sum_{B} g_{n, \delta}^{\beta} D_{\beta}^{p-1}\left(\frac{p+2}{2}\right)
\end{aligned}
$$




$$
p^{-\left(\frac{n p}{2}+k+a\right)} \Gamma\left(\frac{n p}{2}+k+a\right) f_{p}^{\frac{1}{2} p(p+1)-2+a+g+d}
$$

REMARK 2.

An important observation is that in the special case when $(n-p-1) / 2$ is an integer, the summation over $d$ becomes finite (see Remark 1) in (3.6) which means the noncentral density of $f_{p}=1-l_{p} / l_{1}$ involves on $1 y$ two infinite sums. ACKNOWLEDGMENT. The author wishes to acknowledge the support from Miami University in the form of a Sumer Research Fellowship.

\section{REFERENCES}

1. Constantine, A. G. Some noncentral distribuiton problems in multivariate analysis, Ann. Math. Statist., 34 (1963) 1270-1285.

2. Anderson, T. W. Ann. Math. Statist., 17 (1946) 409-431.

3. James, A. T. Distributions of matrix variates and latent roots derived from no rmal samples, Ann. Math. Statist., 35 (1964) 475-501.

4. Khatri, C. G. and Pillai, K. C. S. On the noncentral distributions of two test criteria in multivariate analysis of variance, Ann. Math. Statist., 39 (1968) 215-226.

5. Krishnaiah, P. R. and Waikar, V. B. Simultaneous tests for equality of latent roots against certain alternatives - II, Aerospace Research Laboratories Technical Report ARL, (1969) 69-0178.

6. Sugiyama, T. On the distribution of the largest latent root of the covariance matrix, Ann. Math. Statist., 38 (1967) 1148-1151.

7. Sugiyama, T. Joint distribution of the extreme roots of a covariance matrix, Ann. Math. Statist., 41 (1970) 655-657.

8. Waikar, V. B. and Schuurmann, F. J. Exact joint density of the largest and smallest roots of the Wishart and MANOVA matrices, Utilitas Mathematica, 4 (1973) 253-260.

9. Wigner, E. P. Random matrices in physics, SIAM Review, 9 (1967) 1-23. 


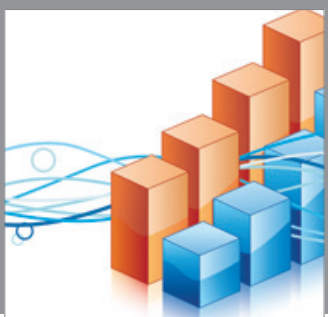

Advances in

Operations Research

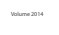

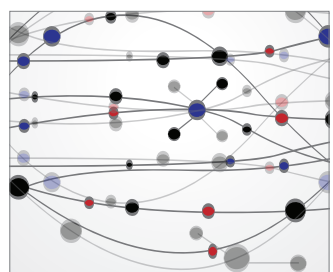

\section{The Scientific} World Journal
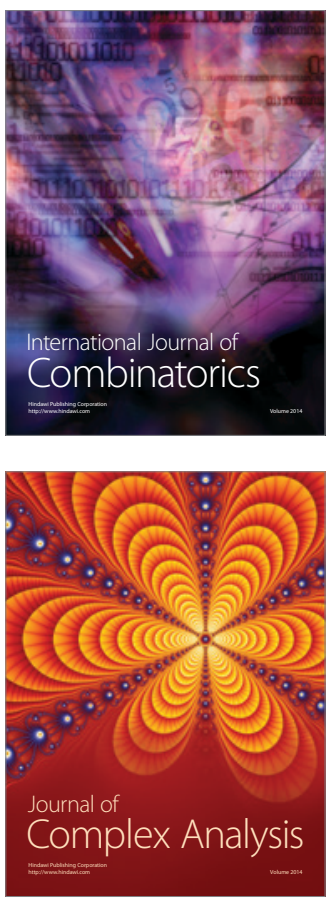

International Journal of

Mathematics and

Mathematical

Sciences
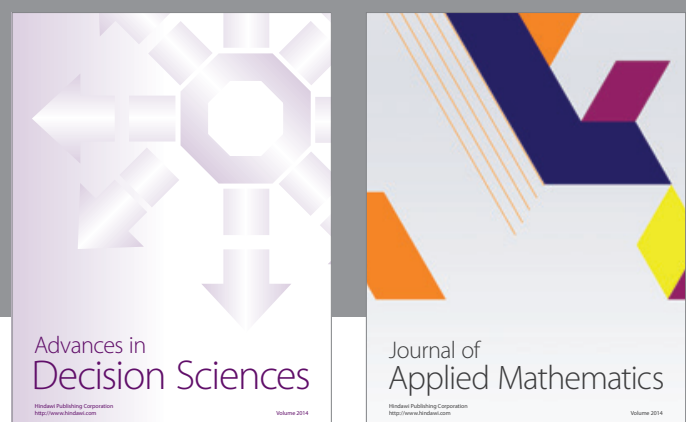

Journal of

Applied Mathematics
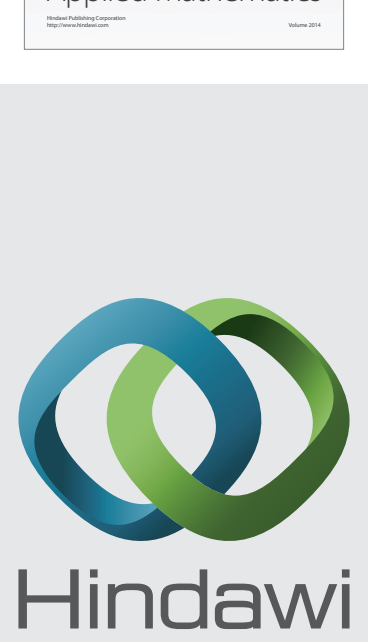

Submit your manuscripts at http://www.hindawi.com
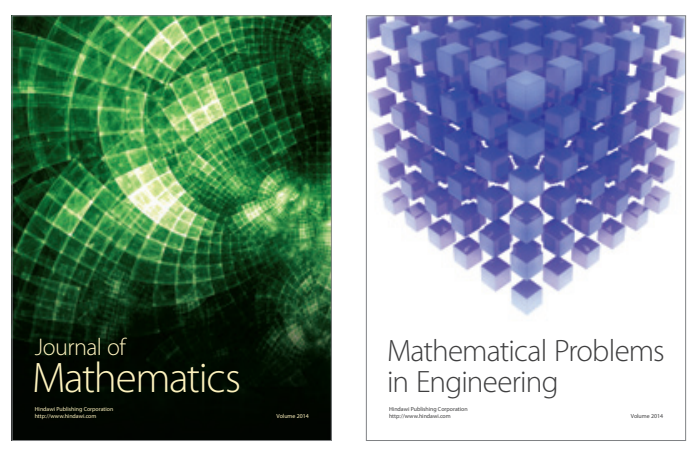

Mathematical Problems in Engineering
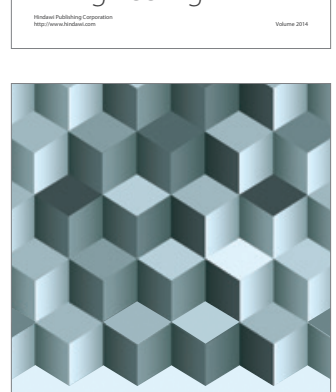

Journal of

Function Spaces
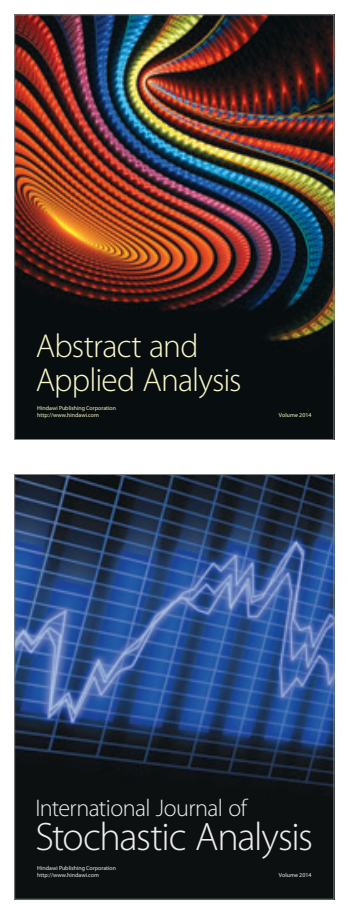

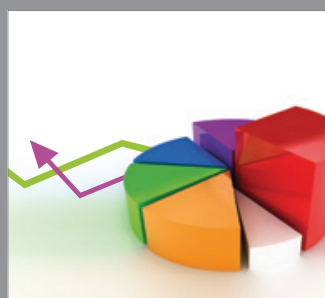

ournal of

Probability and Statistics

Promensencen
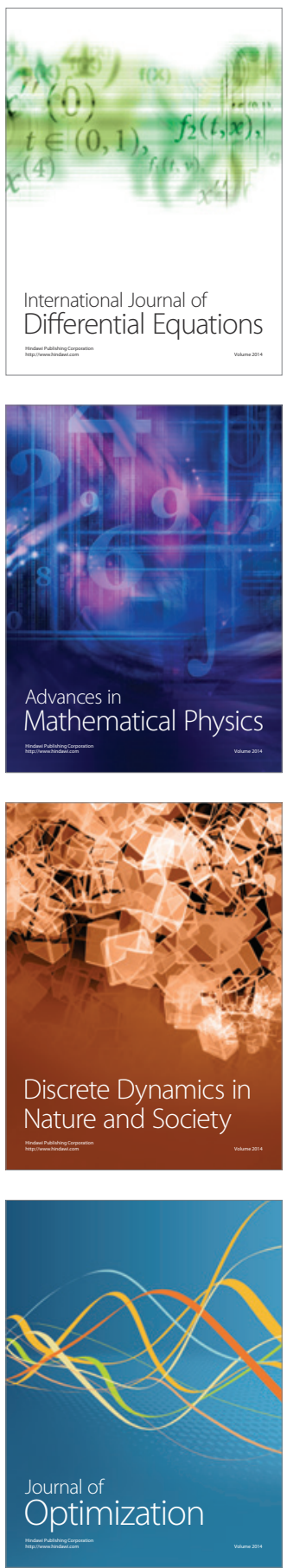